\title{
Asymmetric biasing for subgrid pattern adjustment
}

\author{
Alfred K. Wong ${ }^{a}$ and Lars W. Liebmann \\ IBM Semiconductor Research and Development Center \\ 2070 Route 52, Hopewell Junction, NY 12533, USA \\ ${ }^{a}$ currently at Department of Electrical and Electronic Engineering \\ The University of Hong Kong, Pokfulam Road, Hong Kong
}

\begin{abstract}
The location of a printed edge can be controlled to a fineness that is two orders of magnitude smaller than the design grid, if a slight displacement of the pattern can be tolerated. The essence of this asymmetric subgrid biasing technique is the crenelation of two edges of a pattern into different periods. Fractional arithmetic results in a bias increment that much smaller than that can be achieved with halftone biasing. For a design grid of $20 \mathrm{~nm}(1 X)$, and an exposure system with $\lambda=248 \mathrm{~nm}, N A=0.68$, and $\sigma=0.8$, the bias increment can be as small as $0.22 \mathrm{~nm}$.
\end{abstract}

Keywords: optical lithography, optical proximity correction, halftone biasing, asymmetric subgrid biasing

\section{INTRODUCTION}

As circuit dimension shrinks the mask design grid $(\Delta)$ decreases accordingly. The need to tightly control across-chip linewidth variation (ACLV) by optical proximity correction (OPC) ${ }^{1-6}$ puts further pressure on the design grid. OPC by biasing is direct and effective ${ }^{7}$; but the adjustment fineness is limited by the grid size $\Delta$. Suppose a mask feature of nominal effective dimension $W^{*}$, as shown in Fig. 1(a), needs to be widened, the amount of widening is not arbitrary. It is restricted to integer multiples of $\Delta$. With the least amount of bias $(\Delta)$, the printed pattern has a width of $W+\Delta$ (assuming a linear replication process), and the center of the printed pattern is shifted by $\Delta / 2$. If no pattern placement error should be introduced by biasing, the original feature must be biased equally on both edges, resulting in a bias increment of $2 \Delta$, as shown in Fig. 1(b). In the printing of small features such that the mask error factor $(\mathrm{MEF})^{8}$ is greater than unity, the bias fineness is further reduced by the mask error factor. ${ }^{\dagger}$ For a gate CD control requirement of $5 \mathrm{~nm}$ for the $70 \mathrm{~nm}$ generation, the design grid approaches $1 \mathrm{~nm}(1 X) .^{9}$

A small design grid decreases mask-making throughput. Using raster-scanned exposure tools with traditional writing techniques, a mask with $200 \mathrm{~nm}$-DRAM patterns and a $10 \mathrm{~nm}(1 X)$ grid size takes 20 hours to write. ${ }^{10}$ The mask-writing time increases quadratically with decreasing design grid. Extrapolation to a $1 \mathrm{~nm}$ grid means that a mask takes 2000 hours to write. The multipass gray (MPG) ${ }^{11}$ writing method improves throughput by a factor of four; but the quadratic dependence remains. For raster-scan maskwriting systems, it is desirable to write a mask with a larger design grid $(\Delta)$ and achieve the effect of a smaller grid size.

E-mail: A. K.W.—awong@eee.hku.hk; L.W.L.-lliebman@us.ibm.com

${ }^{*}$ The effective mask feature size is defined as the reticle dimension divided by the exposure system reduction factor. All sizes in this paper refer to the $1 X$ effective dimension.

${ }^{\dagger}$ A mask error factor of one is assumed in this paper. 


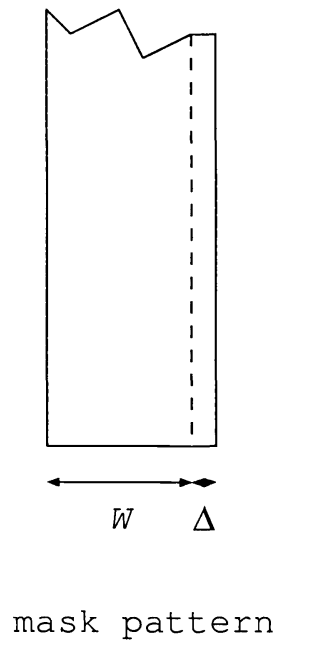

(a) pattern shift

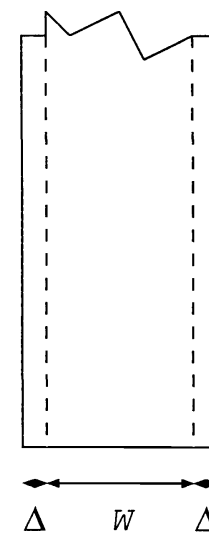

mask pattern

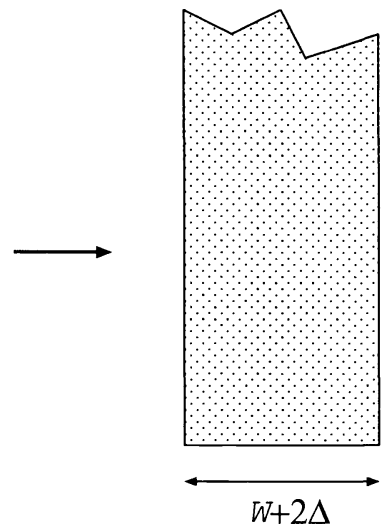

printed image

Figure 1. The fineness of biasing is limited by the grid size $\Delta$.

\section{HALFTONE BIASING}

The halftone technique ${ }^{12}$ can be used to relax pixel size limitations. The method is analogous to halftone printing. ${ }^{13}$ Since the exposure system acts as a low-pass filter, the theoretical pitch resolution limit is ${ }^{14}$

$$
p_{\min }=\frac{\lambda}{N A(1+\sigma)}
$$

where $\lambda$ is the illumination wavelength, $N A$ is the numerical aperture, $\sigma$ is the partial coherence factor, and $p_{\min }$ is the theoretical minimum pitch resolvable by the exposure system. Features with spatial periods less than $\frac{\lambda}{N A(1+\sigma)}$ are not resolved; only the average transmittance is imaged. Suppose an edge of a line is segmented, as shown in Fig. 2, into units of period $n \Delta$ on the reticle, the detail crenelation of this edge is not observed in the image. Only the fill ratio $\frac{a}{n}$ is transmitted, resulting in a feature wider by $\frac{a}{n} \Delta$. The edge can thus be biased in increments of

$$
\Delta_{\mathrm{app}}=\frac{\Delta}{n}
$$

where $\Delta_{\text {app }}$ is the apparent grid size. For biasing without introduction of placement shift, the apparent grid size is $\frac{2 \Delta}{n}$.

The bias increment becomes finer by increasing $n$. But the apparent grid cannot be made arbitrarily small because the exposure system begins to resolve the small pixels when $n$ becomes too large. To hide the details of the crenelation, the segmentation period $(n \Delta)$ must be less than the limit expressed in Eq. (1). The upper-bound $n_{\max }$ is

$$
n_{\max }=\left[\frac{\lambda}{N A(1+\sigma)} \frac{1}{\Delta}\right]
$$

where the square brackets denote the largest integer not exceeding the value of the delimited expression. For a process with $\lambda=248 \mathrm{~nm}, N A=0.68, \sigma=0.8$, and $\Delta=20 \mathrm{~nm}$, Eq. (2) says $n_{\max }=10$. A bias increment $\left(\Delta_{\text {app }}\right)$ of $2 \mathrm{~nm}$ can be achieved with a design grid of $20 \mathrm{~nm}$. If the center of the pattern should not shift, the achievable bias increment doubles to $4 \mathrm{~nm}$ because both edges of the pattern must be adjusted by the same amount. 

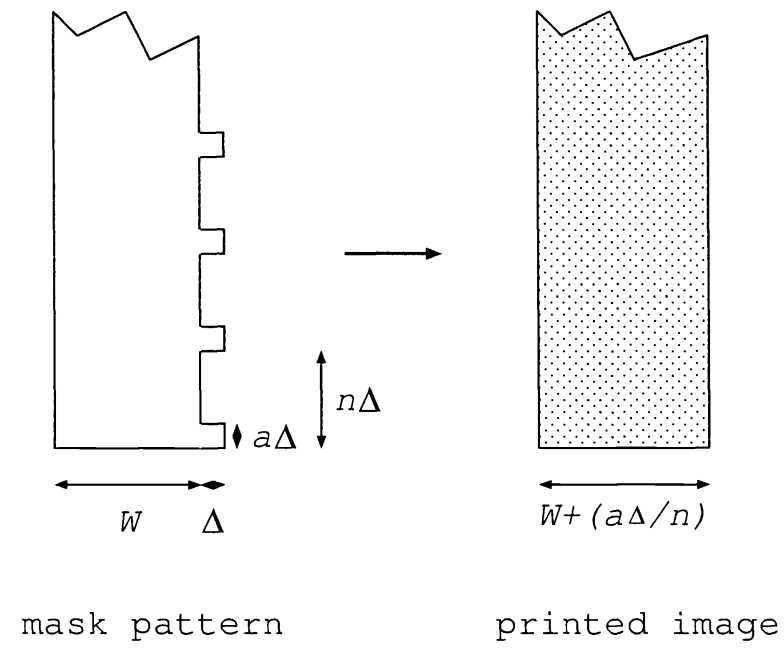

Figure 2. Location of a printed edge can be better controlled by the halftone technique.

\section{ASYMMETRIC SUBGRID BIASING}

The ability to reduce the apparent grid to a tenth of the design grid is an improvement, but further refinement can be made if a slight displacement of the pattern is tolerable. If the two edges of a line are segmented into different periods, an apparent grid much finer than that can be individually achieved is possible. Let us illustrate by the example shown in Fig. 3. The mask feature is biased by segmenting the left edge with a period of $2 \Delta\left(n_{\text {left }}=2\right)$ and the right edge with a period of $5 \Delta\left(n_{\text {right }}=5\right)$. In Fig. 3(a), the left edge is crenelated by writing one pixel out of two, giving a fill ratio of $1 / 2$. The fill ratio of the right edge is $1 / 5$. As a result, the edge is biased wider by

$$
\frac{1}{2} \Delta+\frac{1}{5} \Delta=\frac{7}{10} \Delta
$$

To narrow the feature by $\Delta / 10$, the edges can be crenelated according to Fig. $3(\mathrm{~b})$. The bias increment is $\Delta / 10$.

In the general situation, as shown in Fig. 4, where the right edge is segmented with a pitch of $n_{\text {right }} \Delta$ and a fill ratio of $a / n_{\text {right }}$, and the left edge has a crenelation period of $n_{\text {left }} \Delta$ and a fill ratio of $b / n_{\text {left }}$, the edge is biased by

$$
\frac{\left(b \times n_{\text {right }}+a \times n_{\text {left }}\right)}{n_{\text {right }} \times n_{\text {left }}} \Delta .
$$

Because of fractional arithmetic, such segmentation results in an apparent grid of

$$
\Delta_{\text {app }}=\frac{\Delta}{\left[n_{\text {left }}, n_{\text {right }}\right]}
$$

where the square brackets denote the least common multiple (LCM) of $n_{\text {left }}$ and $n_{\text {right }}$. If $n_{\text {left }}$ and $n_{\text {right }}$ are relative primes,

$$
\Delta_{\text {app }}=\frac{\Delta}{n_{\text {left }} \times n_{\text {right }}}
$$

Note that with this type of biasing, the center of the pattern will shift in general. 


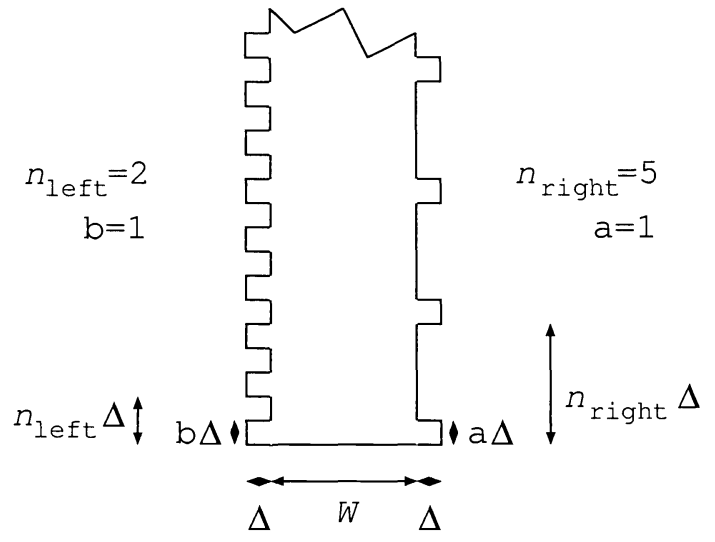

mask pattern

$$
\begin{aligned}
\mathrm{CD} & =W+\frac{1}{2} \Delta+\frac{1}{5} \Delta \\
& =W+\frac{7}{10} \Delta
\end{aligned}
$$

(a)

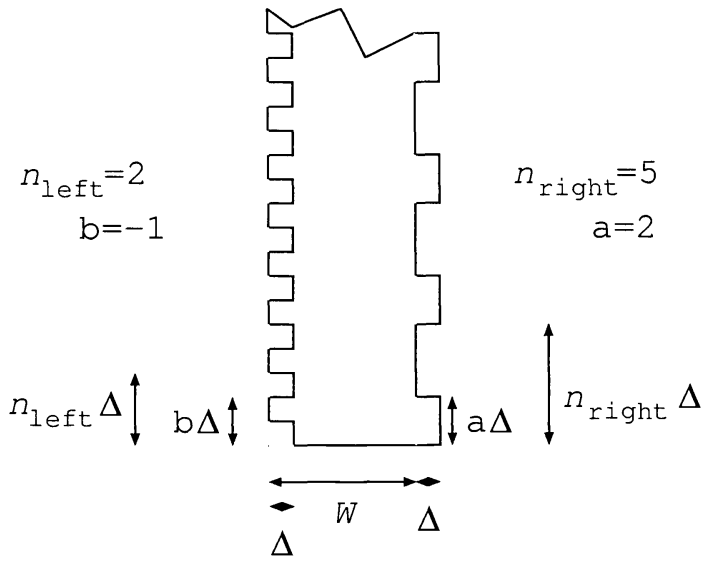

mask pattern

$$
\begin{aligned}
\mathrm{CD} & =W-\frac{1}{2} \Delta+\frac{2}{5} \Delta \\
& =W-\frac{1}{10} \Delta
\end{aligned}
$$

on the right.

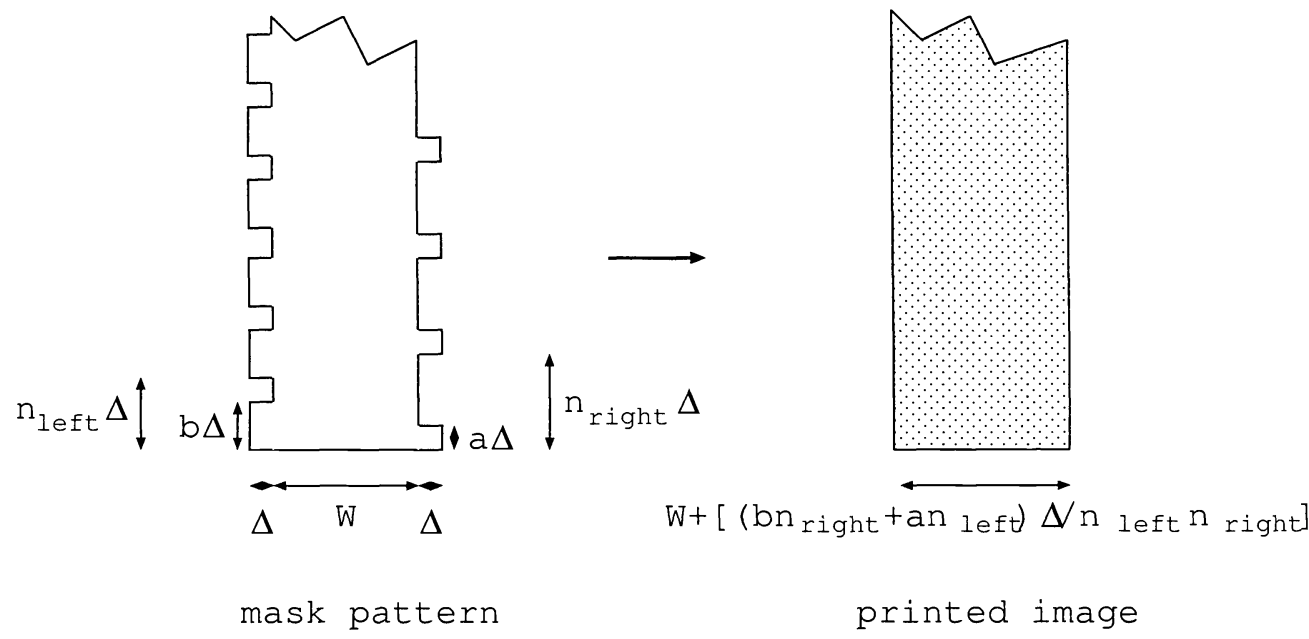

Figure 4. Asymmetric subgrid biasing further reduces the apparent grid. 


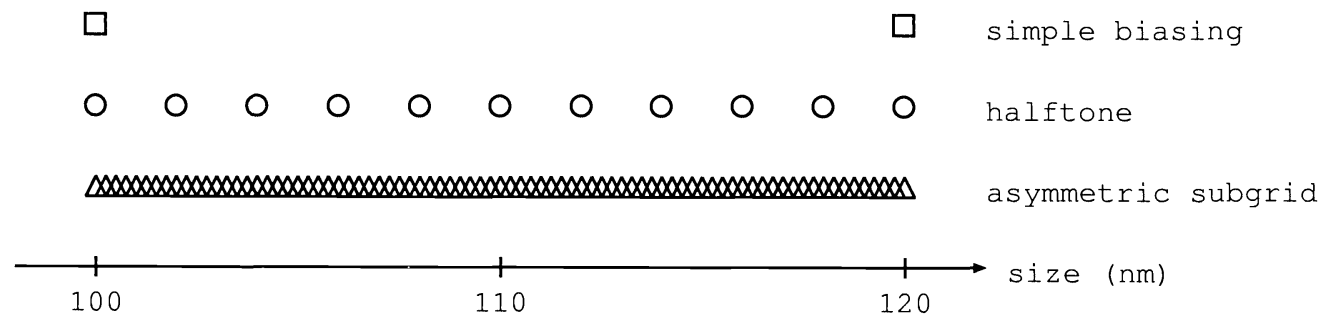

Figure 5. The achievable nominal dimensions between $100 \mathrm{~nm}$ and $120 \mathrm{~nm}$ using different biasing techniques. A design grid of $20 \mathrm{~nm}$, an exposure system with $\lambda=248 \mathrm{~nm}, N A=0.68$, and $\sigma=0.8$ are assumed.

Table 1. Capabilities of three biasing techniques assuming a design grid of $\Delta$.

\begin{tabular}{c|cc} 
& $\Delta_{\text {app }}$ & $\Delta_{\text {app }}$ \\
Technique & (no displacement) & (shift allowed) \\
\hline simple biasing & $2 \Delta$ & $\Delta$ \\
halftone biasing & $2 \Delta / n_{\max }$ & $\Delta / n_{\max }$ \\
asymmetric subgrid biasing & $\mathrm{N} / \mathrm{A}$ & $\Delta /\left[n_{\max } \times\left(n_{\max }-1\right)\right]$
\end{tabular}

Given a value of $n_{\max }$, what is the finest bias increment? Since $n_{\max }$ and $\left(n_{\max }-1\right)$ are relative primes, the smallest apparent grid of asymmetric subgrid biasing is

$$
\Delta_{\mathrm{app}}=\frac{\Delta}{n_{\max } \times\left(n_{\max }-1\right)} .
$$

Using the same parameters as above $\left(\lambda=248 \mathrm{~nm}, N A=0.68, \sigma=0.8, \Delta=20 \mathrm{~nm}\right.$, and $\left.n_{\max }=10\right)$, a bias increment as small as

$$
\Delta_{\text {app }}=\frac{\Delta}{90}=0.22 \mathrm{~nm}
$$

can be achieved. The design grid $\Delta$ and the apparent grid $\Delta_{\text {app }}$ differs by almost two orders of magnitude.

\section{TECHNIQUES COMPARISON}

The capabilities of the three biasing techniques discussed above are listed in Table 1. If pattern correction should not introduce any placement error, simple biasing gives a $2 \Delta$ increment, and the halftone method results in a $2 \Delta / n_{\max }$ control. Asymmetric subgrid biasing is not applicable. If a slight pattern shift is allowed, the apparent grids for simple and halftone biasing are halved, and the fineness of asymmetric subgrid biasing is $\frac{\Delta}{n_{\max } \cdot\left(n_{\max }-1\right)}$. The difference between these techniques is illustrated graphically in Fig. 5 .

\section{DISCUSSION}

Asymmetric subgrid biasing offers superior biasing capability compared with existing techniques. Similar to other resolution enhancement techniques, however, there are many technical and logistic issues to address before the method can be implemented in manufacturing. Writing the fine pixels, as well as mask metrology and inspection, are problems that need to be solved. Logistics such as data volume and algorithms for efficient implementation are also concerns. The established OPC infrastructure can serve as the foundation for its implementation. 


\section{ACKNOWLEDGMENTS}

The authors are grateful for input from Richard Ferguson and Anne McGuire.

\section{REFERENCES}

1. M. D. Prouty and A. R. Neureuther, "Optical imaging with phase shift masks," in Proc. SPIE, vol. 470, pp. 228-232, 1984.

2. J. P. Stirniman and M. L. Rieger, "Fast proximity correction with zone sampling," in Proc. SPIE, T. Brunner, ed., vol. 2197, pp. 294-301, 1994.

3. O. Otto and R. Henderson, "Advances in process matching for rules-based optical proximity correction," in Proc. SPIE, vol. 2884, pp. 425-434, 1996.

4. N. Cobb and A. Zakhor, "Experimental results on optical proximity correction with variable threshold resist model," in Proc. SPIE, G. Fuller, ed., vol. 3051, pp. 458-468, 1997.

5. J. F. Chen, T. Laidig, K. Wampler, and R. Caldwell, "Optical proximity correction for intermediate-pitch features using sub-resolution scattering bars," J. Vac. Sci. Technol. B 15, pp. 2426-2433, Nov. 1997.

6. A. K. Wong, Resolution Enhancement Techniques in Optical Lithography, chapter 4, pp. 91-115. SPIE Press, 2001.

7. L. Liebmann, B. Grenon, M. Lavin, and T. Zell, "Optical proximity correction: a first look at manufacturability," Microlithography World 4(2), pp. 7-11, 1995.

8. A. Wong, R. Ferguson, and S. Mansfield, "The mask error factor in optical lithography," IEEE Transactions on Semiconductor Manufacturing 13, pp. 235-242, May 2000.

9. The National Technology Roadmap for Semiconductors, pp. 82-98. 1997.

10. R. Dean, D. Alexander, J. Chabala, T. Coleman, C. Hartglass, M. Lu, C. Sauer, and S. Weaver, "Investigation of lithography performance using multipass gray (MPG) with MEBES 5000," in Proc. SPIE, U. Behringer, ed., vol. 3665, pp. 166-178, 1999 .

11. F. Abboud, D. R., J. Doering, W. Eckes, M. Gesley, U. Hofmann, T. Mulera, R. Naber, M. Pastor, W. Phillips, J. Raphael, R. Raymond, and C. Sauer, "Multipass gray printing for new MEBES 4500S mask lithography system," in Proc. SPIE, N. Aizaki, ed., vol. 3096, pp. 116-124, 1997.

12. K. H. Nakagawa, F. Chen, R. Socha, T. Laidig, K. E. Wampler, D. van den Broeke, M. Dusa, and R. Caldwell, "Halftone biasing OPC technology: An approach for achieving fine bias control on raster-scan systems," in Proc. SPIE, vol. 3748, pp. 315-323, 1999.

13. P. G. Engeldrum, "Color gamut limits of halftone printing with and without the paper spread function," Journal of Imaging Science and Technology 40, pp. 239-244, May 1996.

14. A. K. Wong, Resolution Enhancement Techniques in Optical Lithography, section 2.3, pp. 45-54. SPIE Press, 2001. 